\title{
Intelligent Surveillance Robot for Monitoring Outdoor Security
}

\author{
Aswin Vishal Kumar ${ }^{1}$ \\ Information Technology, \\ PSG Ploytechnic, Tamil Nadu, India \\ ${ }^{2}$ Kiran \\ Information Technology, \\ PSG Ploytechnic, Tamil Nadu, India
}

\author{
${ }^{3}$ Sandeep \\ Information Technology, \\ PSG Ploytechnic, Tamil Nadu, India \\ ${ }^{4}$ Santhiya \\ Lecturer, Information Technology, \\ PSG Ploytechnic, Tamil Nadu, India
}

\begin{abstract}
This paper presents a new security solution that integrates vision, intelligent algorithm and robot technology. The solution can be applied to guarding large facilities, critical infrastructures and national borders. A human guard can be replaced with such a robot system to take care of dangerous surveillance tasks. Overall structure of the system and description of each component are presented. This project presents a modern approach for surveillance using a Robot. The surveillance system is one which is used for the purpose of security.This system is designed to develop a video monitoring, capturing the image and to store video frames for further verification. Video surveillance is the process of monitoring a situation, an area or a person. The robot is controlled sitting at a far secure place and safely devise a plan to tackle their activities it acquires image from cameras through a web browser, wifi or a mobile application.It receives real-time uploaded image from cameras.
\end{abstract}

KeyWords: Intelligent algorithm, Robot technology, Critical infrastructures, Human guard, Dangerous surveillance

\section{INTRODUCTION}

Recent growth in the business is taking place not only in quantity but also in quality. Some of those examples are high resolution cameras, video signals over the Internet, and advancements in video analytics. This paper presents a new approach of technological improvements in security solution for large facilities or national borders, which is an integration of security devices with intelligence and robot technology.

Conventional CCTV security solution is based upon CCTV cameras, DVRs (Digital Video Recorders), and TV monitors. A group of human operators is responsible to find out an abnormal situation, while looking over live camera images displayed on a series of TV monitors, and takes a counter activity. Some modernized systems use CMS (Central Monitoring System) software to display live images on PC monitors, and users can change the arrangement of images and incorporate IR sensors, which are typically used to provide an intrusion alarm. Some of the cameras are mounted on pan-tilt devices and human operators can select and move a camera to a desired position using a keypad and a joystick.

In such an environment, operators are prone to failure to detect an abnormal situation due to human factors, and recorded images from DVRs were mostly referred only incident to search for clues or evidences for tracking

This project presents a modern approach for surveillance using a Robot. The surveillance system is one which is used for the purpose of security. This system is designed to develop a video monitoring, capturing the image and to store video frames for further verification. Video surveillance is the process of

monitoring a situation, an area or a person. The robot is controlled sitting at a far secure place and safely devise a plan to tackle their activities it acquires image from cameras through a web browser, wifi or a mobile application. It receives real-time uploaded image from cameras. This robot has the ability detects metal bombs, fire using a sensor. Wireless camera mounted on the robot provides us continuous streaming of the defined outdoor area and a stepper motor is used for the rotation of the wireless camera. Surveillance is done even in complete darkness by using Infrared lighting. IR detector is used for the obstacle detection. Two DC motors are interfaced at the receiving end of the microcontroller, which controls the movement of the robotic vehicle. GSM module is used to get the message about moving object. The Robot can be controlled by both manual and automatic mode. This robot replaces human being for security purpose. criminals or intruders. Even if an operator successfully detects an event instantly, time delay is inevitable for intercepting an intruder by dispatching a counter response unit to the location.

\section{SYSTEM DESIGN AND DEVICE}

TSM (Total Surveillance Manager) is an intelligent surveillance and security robot system, which is designed for monitoring behaviors and activities in a wide area utilizing multiple types of surveillance devices including robots, CCTV cameras, ground surveillance radars

\subsection{Integrated Control Environment}

The system consists of Android smart phone, laptop and various sensors, Raspberry Pi board and. Raspberry Pi is connected to the USB camera with the help of USB port .In the PC, Raspbian operating system is installed. Raspberry- pi works only on Raspbian Linux operating system. One can control the robot from remote end say mobile or laptop with the use of Wi-Fi as well as Internet 
and also we can get the live streaming of video from the robot for the purpose of surveillance, this video is obtained on web browser of the remote device from where we are operating the device also we are able to control the robotic movement. DC motors are being used for the movement of robotic wheels and stepper motor is used for camera movement. Raspberry $\mathrm{Pi}$ is used for video processing and sending the processed video to user PC with the help of Wi-Fi as well as internet. A PC can be hacked and files can be erased while this system can overcome those problems. In this system an authentication is required on user side inorder to view the streamed video in the browser. Wide area can be captured. In manual mode the robot can be controlled manually some functions such as controlling the movement. In automatic mode it surrounds the allotted area and report when a moving objected detected and in case of fire.

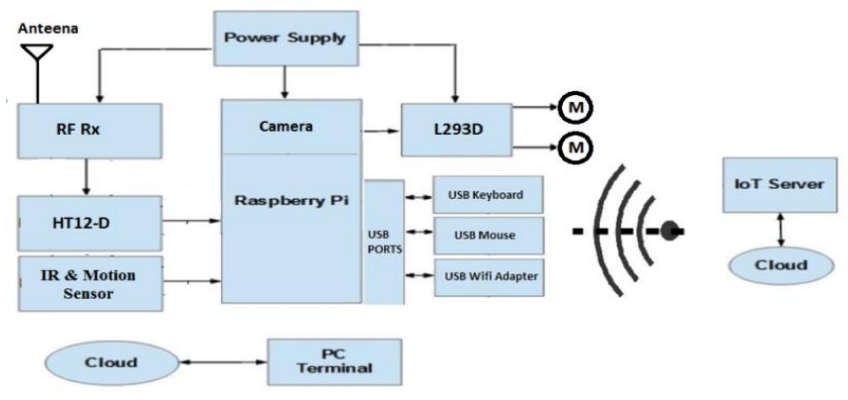

fig -1: Working of Surveillance and Security Robot System

\subsection{Surveillance Guard Robot}

It is largely composed of two modules, the tracking module and the detection module. While the detection module is composed of CCD and thermal video cameras for day and night time use, the tracking

target detected by intelligent cameras or ground surveillance radars based on intelligent algorithms. A lethal weapon such as rifles, a non-lethal acoustic weapon, or a high power halogen lamp can be installed at the tracking module for suppression of targets.

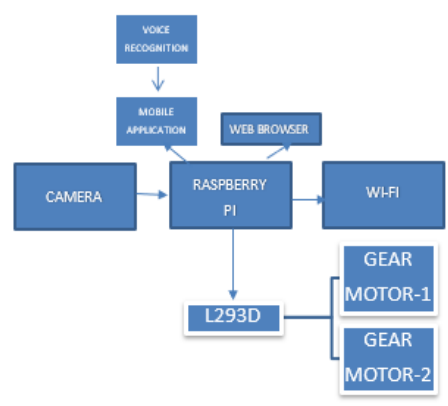

fig -2: Block Diagram Of The Robot System

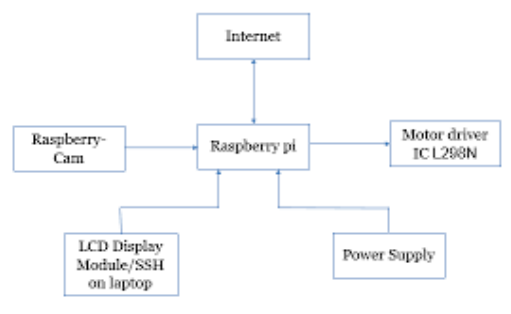

fig -3: Working Of Camera For Obstical Detection

\subsection{Intelligent Camera}

In day time, CCD camera is usually better because it provides VGA quality color images. However, at night or in foggy weather, thermal camera is more suitable since it is not affected by ambient light. Switching from CCD camera to thermal camera or vice versa can be controlled which is adjustable by operators.

Operators can move the camera horizontally and vertically and adjust the optical zoom level. Intelligent cameras send real time video to Digital Video Recorder (DVR) and Video Analytic Devices (VA) over optical link. Consoles can display live videos from DVR over TCP/IP. On the other hand, VAs analyze live videos and detect moving targets

\subsection{Ground Surveillance Radar}

Ground surveillance radars are used to detect moving objects in a wide open and flat area.

\subsection{Fire Alarm Camera and ANPR Camera}

Fire alarm cameras are conceptually similar to intelligent cameras, but its VA algorithms are different from that of intelligent cameras. While VA for intelligent camera is to detect solid moving objects and ignore movements of natural objects such as tree branches, snow or rain, VA for fire alarm is to detect flames and smokes and ignore solid moving objects. ANPR cameras and the ANPR server recognize license plates of vehicles passing at important check points, such as gates

\section{CONCLUSION}

In this paper, an intelligent surveillance and security solution that incorporates robots is presented. Robots reduce risks for humans The implementation of this project to resolve the problem of replacing human to surveillance robot, because of this reduce harm of human resources.

The benefit of robots has increased their flexibility with being capable of performing a variety of tasks and applications. They are more precise and consistent than human workers. Robots also allow for increased production and profit margin because they can complete tasks faster.

\section{ACKNOWLEDGEMENT}

We thank our faculty guide Ms.K.Santhiya, Lecturer Department of Information Technology, PSG Polytechnic College for her constant support and encouragement throughout this mini project work. We are grateful to have 
her inspired guidance and invaluable suggestions which motivated us right from the beginning of the mini project work.

\section{REFERENCES}

[1] Final Report mobile robot for surveillance, Emerald Group, ,2011.

[2] Smart Surveillance Robot for Real-Time Monitoring and Control System in Environment and Industrial Applications ,2018.

[3] Final Report ${ }^{3}$ ISSRS: Intelligent Surveillance Sentry Robot System'(in Korean), Korea Ministry of Knowledge Economy, 2009. 